\title{
Ensinar Estatística: Uma Revisão Sistemática Sobre A Formação Do Professor
}

\author{
Teaching Statistics: A Systematic Review About The Teacher Formation
}

\author{
Cristiane de Fatima Budek Dias* \\ Universidade Tecnológica Federal do Paraná - UTFPR \\ Caroline Subirá Pereira** \\ Universidade Tecnológica Federal do Paraná - UTFPR \\ Guataçara dos Santos Junior*** \\ Universidade Tecnológica Federal do Paraná - UTFPR
}

\begin{abstract}
Resumo
O objetivo deste artigo é analisar as publicações científicas a respeito da formação do professor da Educação Básica para o ensino de estatística no cenário mundial. Para tanto, recorreu-se a uma revisão sistemática da literatura, tendo como base a Methodi Ordinatio, a qual permite a ordenação das publicações por relevância. A busca dos artigos foi realizada nas bases de dados: Science Direct, Scielo, Scopus e Web of Science. Para esta análise foram considerados os 15 trabalhos mais relevantes, conforme a classificação dada pelo InOrdinatio. Os resultados dão indícios de que os professores da Educação Básica precisam de conhecimentos que envolvem o currículo, o conteúdo, as estratégias de raciocínio dos estudantes, o contexto e metodologias que favoreçam as práticas em estatística.
\end{abstract}

Palavras-chave: Formação de Professores; Ensino de Estatística; Revisão Sistemática.

\begin{abstract}
The aim of this article is to analyze the scientific publications about the formation of basic education teacher for the teaching of statistics in the world scenario. For this, a systematic review of the literature was used, based on Methodi Ordinatio, which allows the ordering of publications by relevance. The search for articles was carried out in the databases: Science Direct, Scielo, Scopus and Web of Science. For this analysis were considered the 15 most relevant works, according to the classification given by InOrdinatio. The results point that teachers of basic education need knowledge that involves the curriculum, content, students' reasoning strategies, context and methodologies that favor practices in statistics.
\end{abstract}

Keywords: Teacher Training; Statistics Teaching; Systematic Review.

\footnotetext{
* Mestra em Ensino de Ciência e Tecnologia pela Universidade Tecnológica Federal do Paraná (UTFPR). Doutoranda do Programa de Ensino de Ciência e Tecnologia na Universidade Tecnológica Federal do Paraná (UTFPR), Ponta Grossa, Paraná, Brasil. E-mail: cristianed@alunos.utfpr.edu.br

** Mestra em Ensino de Ciência e Tecnologia pela Universidade Tecnológica Federal do Paraná (UTFPR). Doutoranda do Programa de Ensino de Ciência e Tecnologia na Universidade Tecnológica Federal do Paraná (UTFPR), Ponta Grossa, Paraná, Brasil. E-mail: carolinepereira@alunos.utfpr.edu.br

${ }^{* * *}$ Doutor em Ciências Geodésicas pela Universidade Federal do Paraná (UFPR). Docente do Programa de PósGraduação em Ensino de Ciência e Tecnologia da Universidade Tecnológica Federal do Paraná (UTFPR), Ponta Grossa, Paraná, Brasil. E-mail: guata@utfpr.edu.br
} 


\section{Introdução}

A estatística está presente no currículo da Educação Básica de muitos países desde a década de 1980 e assume relevância por se tratar de um campo expressivo nas atividades sociais, políticas e econômicas e nas mais diversas áreas de conhecimento. Há então, uma preocupação com a formação dos estudantes, de modo que sejam capazes de entender o processo de uma investigação. Esse entendimento é fundamental para que possam analisar, questionar e tomar decisões com base em dados quantificados.

Tais colocações sugerem que haja uma formação adequada do professor para esse ensino, pois, caso contrário, a formação dos estudantes poderá ser prejudicada (Costa, Pinheiro \& Costa, 2016). Neste sentido, entende-se que a formação de professores que irão ensinar estatística, demanda por experiências específicas e isso se dá com um atendimento às particularidades que a caracterizam. Além disso, é preciso atender a questões próprias do ensino, ou seja, da didática da estatística.

Deste modo, tem-se como perguntas de partida para este estudo: como as pesquisas que tratam da formação de professores para o ensino de estatística abordam os conhecimentos necessários para o ensino e quais os direcionamentos para uma boa formação docente?

Partindo desses questionamentos, este artigo tem como objetivo analisar as publicações científicas a respeito da formação do professor da educação básica para o ensino de estatística, no cenário mundial, de modo a entender os conhecimentos necessários e os modelos de formação mais adequados, bem como de observar as lacunas existentes no campo de pesquisa. Para tanto, recorreu-se a uma revisão sistemática da literatura, tendo como base metodológica a Methodi Ordinatio, a qual permite uma busca sistematizada e posterior ordenação das publicações por relevância.

\section{Procedimentos Metodológicos}

Para a concretização deste estudo seguiu-se os nove passos da Methodi Ordinatio, que consiste em uma metodologia de seleção e ranqueamento de produções científicas de acordo com sua relevância para o tema de estudo. É uma metodologia multicritérios, já que considera o fator de impacto do periódico, o número de citações e o ano de publicação do artigo (Pagani, Kovaleski, Resende, 2015).

O ranqueamento dos artigos é feito com base no índice de resultado da equação do 
InOrdinatio, sendo que, quanto mais elevado for o índice maior será considerada a relevância científica do conteúdo do artigo. A equação para determinar esse índice é:

Na qual: Fi é o fator de impacto do periódico em que o artigo foi publicado; $\alpha$ corresponde a um valor que varia de 1 a 10, conforme a relevância ao ano de publicação atribuído pelo próprio pesquisador ; e Ci é o número de citações do artigo (Pagani et al., 2015).

A Methodi Ordinatio é composta por 9 passos: 1) definição da intenção de pesquisa; 2) pesquisa preliminar nas bases de dados; 3) definição das palavras-chave; 4) busca definitiva nas bases de dados; 5) filtragem dos dados; 6) identificação do fator de impacto e número de citações; 7) aplicação da Equação InOrdinatio; 8) download dos artigos e 9) leitura completa e análise sistemática.

Como primeiro passo da Methodi Ordinatio tem-se a definição da intenção de pesquisa. Neste caso, a de conhecer as publicações científicas, no cenário mundial, a respeito da formação para o ensino de estatística dos professores da Educação Básica.

No segundo passo se realiza uma pesquisa preliminar nas bases de dados, para a definição das palavras-chave que serão utilizadas na busca definitiva. Neste passo foram utilizadas diversas palavras, variações e combinações relacionadas à temática, observando-se quais delas retornavam produções mais próximas da intenção de pesquisa.

No terceiro passo são definidas as palavras-chave, com base no que foi observado na exploração realizada no passo anterior. Nesta pesquisa, foram definidas as combinações: ("statistic* education" AND teach* training); ("statistic* education" AND teach* formation); ("statistic* education" AND teacher* education); ("statistic* teaching" AND teach* training); ("statistic* teaching" AND teach* formation) e ("statistic* teaching" AND teacher* education).

No quarto passo é realizada a busca definitiva dos artigos nas bases de dados. Neste estudo, as bases consideradas pertinentes para a busca foram: Web of Science, Science Direct, Scopus e Scielo, consideradas apropriadas para o campo de pesquisa em ensino. O resultado

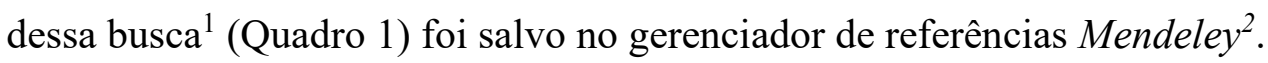

Quadro 1: Resultado da busca

\begin{tabular}{|c|c|c|c|c|c|}
\hline \multirow[b]{2}{*}{ palavras-chave } & \multicolumn{4}{|c|}{ Bases } & \multirow[b]{2}{*}{$\begin{array}{c}\text { Total por } \\
\text { base }\end{array}$} \\
\hline & $\begin{array}{c}\text { Web of } \\
\text { Science }\end{array}$ & $\begin{array}{c}\text { Science } \\
\text { Direct }\end{array}$ & Scopus & Scielo & \\
\hline "statistic* education" AND teach* training & 19 & 14 & 30 & 179 & 242 \\
\hline "statistic* education" AND teach* formation & 5 & 5 & 6 & 55 & 71 \\
\hline
\end{tabular}

\footnotetext{
${ }^{1}$ A busca foi realizada no dia dois de fevereiro de 2019. Essa data é importante, pois uma busca realizada em data posterior, provavelmente retornará outros resultados.

${ }^{2} \mathrm{O}$ Mendeley é um software gratuito que tem por finalidade gerenciar referências (https://www.mendeley.com/).
} 


\begin{tabular}{|l|c|c|c|c|c|}
\hline "statistic* education" AND teacher* education & 55 & 33 & 134 & 399 & $\mathbf{6 2 1}$ \\
\hline "statistic* teaching" AND teach* training & 4 & 4 & 7 & 212 & $\mathbf{2 3 5}$ \\
\hline "statistic* teaching" AND teach* formation & 1 & 2 & 3 & 65 & $\mathbf{7 1}$ \\
\hline "statistic* teaching" AND teacher * education & 26 & 4 & 46 & 183 & $\mathbf{2 5 9}$ \\
\hline
\end{tabular}

Fonte: Elaborado pelos autores

O quinto passo da Methodi Ordinatio consiste na filtragem dos dados retornados na busca definitiva. Nessa filtragem são eliminados os artigos em duplicata e aqueles não relacionados à intenção de pesquisa. A eliminação dos trabalhos duplicados ${ }^{3}$ foi facilitada pelo Mendeley e posteriormente, após leitura dos títulos, resumos e palavras-chave dos artigos, foram eliminados os trabalhos sem relação com ensino de estatística e com a formação do professor da Educação Básica. O número de artigos eliminados nesta etapa está exposto na Tabela 1. Com isso, o portfólio de artigos considerados nesta revisão, totalizou-se em 105.

Tabela 1: Resultado da etapa de filtragem

\begin{tabular}{ccc}
\hline Duplicatas & Desalinhados & Total \\
\hline 693 & $701^{4}$ & $\mathbf{1 0 5}$ \\
\hline
\end{tabular}

Fonte: Elaborada pelos autores

No sexto passo se realiza a identificação do fator de impacto do periódico em que o artigo foi publicado e do número de citações que este apresenta. Foi considerado como fator de impacto o SNIP (Source Normalized Impact per Paper) por se tratar de uma métrica que compara os periódicos de acordo com seus campos temáticos.

Com essas informações, no sétimo passo, aplica-se a Equação InOrdinatio para a ordenação das publicações. Assim, após o levantamento desses números, foi aplicada a equação e se efetuou a ordenação dos artigos do maior para o menor.

O oitavo passo é destinado ao download dos artigos que serão considerados para a leitura completa. Para este estudo, optou-se pela leitura dos primeiros 15 artigos, pois é um número considerado suficiente para análise, tendo em conta a impraticabilidade de leitura, interpretação e discussão da totalidade dos trabalhos. Uma análise que enquadrasse um número superior a 15 artigos resultaria em uma discussão superficial ou voltada para resultados quantitativos, não oportunizando uma visão ampla dos artigos mais relevantes.

\footnotetext{
${ }^{3}$ Alguns artigos constavam em mais de uma das bases, então foram eliminados e permaneceram em apenas uma.

${ }^{4} \mathrm{O}$ número elevado de exclusão de artigos desalinhados pode ser devido às buscas retornarem um grande número de artigos não relacionados com o ensino. Durante a fase de filtragem observou-se que publicações que apresentavam análise estatística de dados e que mencionavam isso no resumo foram carregadas pelas bases, dentre essas, estavam muitas pesquisas na área de saúde. Além disso, muitas pesquisas estavam direcionadas a alunos e professores de cursos de exatas do Ensino Superior.
} 
O Quadro 2 apresenta a codificação (cod.) que será adotada na descrição e análise dos 15 artigos mais relevantes, os autores e títulos, o país de origem do trabalho, o ano de publicação, o SNIP do periódico em que foi publicado, o número de citações e o resultado da Equação InOrdinatio.

Quadro 2: Quinze artigos mais relevantes sobre formação de professores para o ensino de estatística

\begin{tabular}{|c|c|c|c|c|c|c|}
\hline Cod & Autor/Título & País & Ano & SNIP & Citações & InOrdinatio \\
\hline A.01 & $\begin{array}{l}\text { Groth, R.E. Toward a conceptualization of } \\
\text { statistical knowledge for teaching }\end{array}$ & EUA & 2007 & 2,906 & 151 & 141 \\
\hline A. 02 & $\begin{array}{l}\text { Garfield, J.B. and Gal, I. Assessment and statistics } \\
\text { education: current challenges and directions }\end{array}$ & $\begin{array}{l}\text { EUA/ } \\
\text { Israel }\end{array}$ & 1999 & 1,677 & 155 & 105 \\
\hline A.03 & $\begin{array}{l}\text { Roseth, C.J., Garfield, J.B. and Ben-Zvi, D. } \\
\text { Collaboration in learning and teaching statistics }\end{array}$ & $\begin{array}{l}\text { EUA/ } \\
\text { Israel }\end{array}$ & 2008 & 0,746 & 82 & 77 \\
\hline A.04 & $\begin{array}{l}\text { Jacobbe, T. Elementary school teachers' } \\
\text { understanding of the mean and median }\end{array}$ & EUA & 2012 & 1,072 & 51 & 66 \\
\hline A.05 & $\begin{array}{l}\text { Leavy, A. and O'Loughlin, N. Preservice teachers } \\
\text { understanding of the mean: Moving beyond the } \\
\text { arithmetic average }\end{array}$ & Irlanda & 2006 & 1,42 & 70 & 55 \\
\hline A.06 & $\begin{array}{l}\text { Pfannkuch, M., Regan, M., Wild, C. and Horton, } \\
\text { N.J. Telling data stories: essential dialogues for } \\
\text { comparative reasoning }\end{array}$ & $\begin{array}{l}\text { Nova } \\
\text { Zelândia }\end{array}$ & 2010 & 0,746 & 48 & 53 \\
\hline A.07 & $\begin{array}{l}\text { Estrella, S. Comprensión de la media por } \\
\text { profesores de educación primaria en formación } \\
\text { continua }\end{array}$ & Chile & 2016 & 0,53 & 17 & 52 \\
\hline A. $08^{5}$ & $\begin{array}{l}\text { Lopes, C.E. Educação estatística no curso de } \\
\text { licenciatura em matemática }\end{array}$ & EUA/Brasil & 2013 & 0,421 & 29 & 49 \\
\hline A.09 & $\begin{array}{l}\text { Garfield, J. and Everson, M. Preparing teachers of } \\
\text { statistics: A graduate course for future teachers }\end{array}$ & EUA & 2009 & 0,746 & 48 & 48 \\
\hline A. 10 & $\begin{array}{l}\text { Witterholt, M., Goedhart, M. and Suhre, C. The } \\
\text { impact of peer collaboration on teachers' practical } \\
\text { knowledge }\end{array}$ & Holanda & 2016 & 1,417 & 12 & 47 \\
\hline A.11 & $\begin{array}{l}\text { Vetten, A., Schoonenboom, J., Keijzer, R. and } \\
\text { Oers, B. van. The development of informal } \\
\text { statistical inference content knowledge of pre- } \\
\text { service primary school teachers during a teacher } \\
\text { college intervention }\end{array}$ & Holanda & 2018 & 1,702 & 1 & 46 \\
\hline A. 12 & $\begin{array}{l}\text { Gould, R., Bargagliotti, A. and Johnson, T. An } \\
\text { analysis of secondary teachers' reasoning with } \\
\text { participatory sensing data }\end{array}$ & EUA & 2017 & 0,577 & 6 & 46 \\
\hline A. 13 & $\begin{array}{l}\text { Justice, N., Zieffler, A., Huberty, M.D. and } \\
\text { DelMas, R. Every rose has its thorn: secondary } \\
\text { teachers' reasoning about statistical models }\end{array}$ & EUA & 2018 & 1,177 & 0 & 45 \\
\hline A.14 & $\begin{array}{l}\text { Frischemeier, D. and Biehler, R. Preservice } \\
\text { teachers comparing groups with TinkerPlots-an } \\
\text { exploratory laboratory study }\end{array}$ & Alemanha & 2018 & 0,577 & 0 & 45 \\
\hline A. 15 & $\begin{array}{l}\text { Green, J.L., Smith, W.M., Kerby, A.T., } \\
\text { Blankenship, E.E., Schmid, K.K. and Carlson, } \\
\text { M.A. Introductory statistics: Preparing in-service } \\
\text { middle-level mathematics teachers for classroom } \\
\text { research }\end{array}$ & EUA & 2018 & 0,577 & 0 & 45 \\
\hline
\end{tabular}

${ }^{5} \mathrm{O}$ destaque no Quadro 2 se refere ao único texto brasileiro classificado entre os 15 primeiros artigos. Apesar do estudo ter sido realizado a partir da experiência da autora em curso na Universidade da Geórgia, foi publicado em periódico brasileiro e traz uma reflexão sobre os cursos de Licenciatura em Matemática no Brasil. O texto completo pode ser encontrado em: http://ojs-teste.biblioteca.unesp.br/index.php/bolema/article/view/8285. 


\section{Fonte: Elaborado pelos autores}

No nono passo se realiza a leitura completa e a análise sistemática. Durante a leitura completa percebeu-se que os artigos apresentavam três características principais: discussões teóricas, de documentos e de aplicações práticas. Deste modo, para a descrição e análise sistemática, os artigos foram agrupados em três eixos: Discussões Teóricas, Discussões Aplicadas e Discussões Documentais.

\section{Análise Dos Resultados}

Os artigos teóricos trazem discussões conceituais, como por exemplo, dicussões a respeito do conhecimento para ensinar estatística, sobre a avaliação da aprendizagem, entre outros. Esses estudos não se referem à pesquisas realizadas em campo, ou seja, não foram aplicados cursos, questionários ou algum outro instrumento de coleta de dados.

Os artigos de pesquisa aplicada são análises de resultados a partir de aplicações com professores. Nesses estudos há uma interação em campo com professores em formação inicial ou continuada e há a aplicação de cursos, questionários ou entrevistas.

As publicações documentais correspondem aos trabalhos que analisam documentos oficiais. Nesses artigos, os autores apresentam e discutem currículos de cursos ou analisam artigos e documentos de pesquisa apresentados por professores em formação.

As próximas subseções apresentam e discutem os artigos, agrupados em cada um dos eixos definidos neste estudo.

\subsection{Discussões Teóricas}

No recorte dos 15 artigos considerados para a leitura completa, cinco são teóricos, A.01, A.02, A.03, A.06 e A.09. Dentre eles, três tratam de questões relacionadas ao conhecimento para o ensino de estatística, que envolvem conhecimentos específicos (A.01 e A.06) e sobre a avaliação (A.02).

O artigo A.02 (1999) traz uma descrição de métodos alternativos de avaliação da aprendizagem estatística. Os autores de A.02, Garfield e Gal (1999) apontam os conhecimentos estatísticos relevantes para a aprendizagem dos estudantes e, a partir disso, pontuam sete métodos para avaliar a aprendizagem: 1) Projetos individuais e em grupo; 2) Portfólios; 3) Mapas conceituais; 4) Críticas às ideias estatísticas ou questões em notícias; 5) Perguntas 
objetivas para avaliar o pensamento de nível superior; 6) Minute papers ${ }^{6}$ e 7) Avaliação do desempenho na solução de problemas estatísticos. Para cada um desses métodos, os autores trazem exemplos de critérios, pontuação e tarefas que podem ser analisadas no processo avaliativo.

Para atender a questões sobre quando usar determinado método, em que situação e como utilizar os resultados das avaliações, os autores apresentam um framework que trazem os componentes: o que avaliar; o objetivo da avaliação, o responsável pela avaliação (alunos/professor), o método a ser utilizado, a ação a ser tomada e o feedback aos estudantes.

Esses componentes indicam a complexidade da avaliação da aprendizagem em estatística e a necessidade de uma reflexão mais profunda do professor a respeito dos objetivos da avaliação, de como será utilizada e analisada para que se chegue ao método mais apropriado para cada contexto. Deste modo, considera-se que este conhecimento sobre a ação avaliativa e seu processo é imprescindível para os professores da Educação Básica, sendo um componente de conhecimento que não pode ficar de fora, quando se discute o que o professor precisa saber para ensinar estatística.

Groth (2007), autor de A.01, busca esboçar uma possível estrutura do conhecimento estatístico para o ensino. Em sua visão, apesar de estatística e matemática compartilharem "um terreno comum" (p. 427) são campos diferentes. Para o autor, a estatística é uma disciplina em si e não um simples ramo da matemática. Deste modo, o conhecimento necessário para ensinar estatística difere daquele para ensinar matemática. A estatística envolve, primordialmente, atividades não-matemáticas, como construir significado para os dados, examinando contexto e a escolha sobre os caminhos mais adequados para responder a determinadas questões de pesquisa estatística.

Groth (2007) trata, então, do conhecimento comum e do conhecimento especializado para ensinar estatística, baseando-se nos quatro componentes da investigação estatística apresentada no Guidelines for Assessment and Instruction in Statistics Education ${ }^{7}$ (GAISE): formular questões; coletar dados; analisar dados e interpretar resultados (Franklin et al., 2007). Para cada um dos componentes da investigação estatística do GAISE, Groth (2007) tem uma estrutura de conhecimentos comuns e especializados, tanto matemáticos, quanto nãomatemáticos. São conhecimentos bastante específicos e que se interligam. Um ponto

\footnotetext{
${ }^{6}$ Conforme a interpretação dos autores a partir da análise do artigo, entende-se que a expressão minute papers corresponde a uma atividade em que o professor distribui, no final de cada aula, pequenos pedaços de papel para os alunos e sugere que os mesmos registrem conceitos que eles compreenderam.

${ }^{7}$ Diretrizes para Avaliação e Instrução de Educação Estatística: conjunto de recomendações para cursos introdutórios de estatística (Ensino Superior) e educação estatística nos anos pré-K-12.
} 
fundamental observado entre os conhecimentos expostos pelo autor diz respeito ao entendimento das estratégias dos estudantes, para a leitura das diferentes representações gráficas, que é um conhecimento matemático especializado e fundamental, tanto para a avaliação, como para a tomada de decisão, conforme as estratégias apresentadas.

No artigo A.01, ainda, há indicações de meios para o desenvolvimento desses conhecimentos, os quais devem estar pautados em cursos com novas configurações, que conforme sugere, primem pelo uso de artefatos da prática de ensino, tais como, trabalhos realizados por estudantes, planos de aula, vídeos e escrita de casos de ensino (narrativas). Ainda assinala que a formação deve considerar o contexto de ensino, pois a aprendizagem do professor precisa estar fundamentada em situações verdadeiras e contextualizadas.

Outro artigo teórico desta revisão é A.03, no qual Roseth, Garfield e Ben-Zvi (2008) tratam do processo de ensino-aprendizagem colaborativo, e da mesma forma Garfield e Gal (1999), autores de A.02, trazem uma perspectiva de colaboração entre os professores que é interessante, de se pensar em termos de formação para todas as etapas educativas.

Roseth, Garfield e Ben-Zvi (2008) indicam alguns passos envolvidos em práticas de ensino colaborativo: tomada de decisão pré-instrucionais; explicação da tarefa e da estrutura colaborativa para os estudantes; acompanhamento e intervenção durante a atividade colaborativa e processamento em grupo. Esses passos indicam que é preciso um planejamento adequado para as tarefas colaborativas, atentando-se para os objetivos que se pretendem atingir em termos de habilidades interpessoais, de grupo e sociais; uma exposição clara de como as atividades serão conduzidas e do papel de cada estudante dentro desse processo.

Os autores de A.03 apontam também para a necessidade de acompanhamento e intervenção adequados durante a realização das atividades, visto que o professor precisa atuar mediando e observando os estudantes na interação com seus pares. Este é um momento oportuno para avaliar os discursos e para buscar entendimento sobre como raciocinam sobre determinados conceitos estatísticos.

Outro ponto interessante abordado por Roseth, Garfield e Ben-Zvi (2008) é a formação de grupos de ensino colaborativos, ou seja, grupos de professores que ensinam estatística. Esses grupos podem ser formados entre professores de uma mesma instituição ou de instituições diferentes e têm o intuito de partilha de experiências, de ideias e de discussão sobre desafios e perspectivas para o ensino de estatística.

A colaboração fornece bases para que se tornem professores melhores, pois oportuniza progresso na elaboração de materiais, de metodologias e formas de avaliação, proporciona 
reflexão sobre a prática, constrói motivação e apoio, constituindo um senso de comunidade, de suporte e de orientação para professores iniciantes (Roseth et al. 2008).

O último artigo teórico desta análise é A.06, o qual os autores Pfannkuch, Regan, Wild e Horton (2010) discutem as histórias dos dados quantitativos e refletem sobre os desafios nas práticas de ensino atuais. Os autores também debatem sobre o que constitui uma boa verbalização da estrutura em gráficos e resumos numéricos, e tenta esclarecer quais conceitos subjacentes devem ser trazidos para o ensino e como. Tais conceitos são: ler e interpretar informações codificadas a partir de gráficos, compreender e verbalizar com amplo repertório conceitual, com atenção à linguagem, invocando pensamentos descritivos e inferenciais, determinando informalmente, se as reivindicações podem ser feitas sobre as populações.

Em A.06 é apresentado um guia destinado ao desenvolvimento dos professores de estudantes entre 14 a 18 anos, idades pertinentes às avaliações realizadas na Nova Zelândia, país em que atuam os autores do artigo. Essas idades correspondem ao Ensino Médio brasileiro, última etapa da Educação Básica. O guia tem foco nas palavras e verbalizações para o raciocínio comparativo, ou seja, comparações entre duas amostras ou conjunto de dados, abordando os conceitos que precisam ser discutidos e a linguagem que deve ser usada para comunicar a infinidade de ideias necessárias para interpretar a plotagem de gráficos.

O guia apresentado pelos autores baseia-se no ciclo de investigação estatística do PPDAC (Problema, Plano, Dados, Análise, Conclusão (Wild \& Pfannkuch, 1999)) e traz notas aos professores, que incluem ensino e técnicas para justificar o uso de imagens e linguagem particulares. Isso porque, ao colocarem o guia no quadro do ciclo PPDAC, os autores fundamentam a comunicação em termos do processo da coleta de dados, desde a formulação da questão sobre as populações até a conclusão, e as ligações entre as fases do ciclo. Com as notas do professor os autores reforçam a lógica de formas particulares de pensar e falar.

As principais questões e estratégias subjacentes ao guia são: o que faz uma boa pergunta? Como se pode destacar e preservar a distinção entre pensamentos descritivos e inferenciais? Quais devem ser as primeiras impressões quando se olha para um enredo? Que papel o conhecimento contextual desempenha? Como se tenta esclarecer algumas questões estatísticas? Qual é o propósito de raciocinar a partir de plotagem de gráficos?

As questões apresentadas por Pfannkuch et al. (2010) são importantes para fundamentar o raciocínio do professor sobre comparações e sobre como desvendar as histórias dos dados, sempre com atenção para o contexto em que são produzidos. 


\subsection{Discussões Aplicadas}

Dentre os 15 estudos mais relevantes desta análise, oito apresentam aplicações práticas em que as percepções dos professores são verificadas ou cursos são descritos como mobilizadores de conhecimentos de conteúdo e pedagógico para o ensino de estatística. Os artigos tratam da mobilização do conhecimento de medidas de tendência central, principalmente da média (A.05, A.04 e A.07), inferência informal (A.11 e A.13), análise exploratória de dados (A.12) e raciocínio comparativo (A.14), tema observado em A.06, descrito anteriormente.

Os artigos A.05, A.04 e A.07 tratam dos conhecimentos de professores do ensino elementar (que corresponde aos anos iniciais do Ensino Fundamental brasileiro) em formação inicial e continuada sobre o conceito de média. Os resultados desses estudos mostram que a compreensão dos professores está atrelada aos procedimentos para o cálculo, em detrimento do entendimento conceitual da medida. Isso revela que há falhas na formação básica dos professores, tanto no contexto nacional como internacional.

Observa-se que os questionários e entrevistas aplicados tanto à professores em exercício, quanto àqueles em formação inicial, guardam semelhanças, como por exemplo, em relação às dificuldades em apresentar argumentos para justificar porque a média seria ou não a medida mais adequada em determinadas situações e o que essas medidas representam dentro de um contexto particular.

Os resultados apresentados em A.07, que tratou do conhecimento sobre a média com dados contendo outliers, trazem concepções corretas e incorretas e revelam que as ideias estatísticas de contexto e representatividade de um conjunto de dados estão distantes da formação dos professores. A autora Estrella (2016), do artigo A.07, revelou que não houve compreensão dos outliers como conceito estatístico e da influência desses sobre a média.

Em A.05, os autores Leavy e O'Loughlin (2006) levantam questionamentos a respeito da compreensão conceitual raramente coexistir com o conhecimento procedimental e critica o fato de os estudantes, assim como os professores, passarem anos na escola básica e não construírem uma compreensão conceitual satisfatória da média. Neste sentido, os autores de A.05, assim como dos demais artigos, (A.04 e A.07) ressaltam que o ponto de partida para a melhoria do ensino é o conhecimento do conteúdo e o conhecimento pedagógico deste conteúdo (Shulman, 1986) por parte dos professores.

O conhecimento pedagógico do conteúdo sobre a média faria com que o professor 
refletisse seu próprio conhecimento sobre o conceito, identificando os principais componentes que devem ter precedência. Isso permitiria que o professor apoiasse a construção da compreensão conceitual, desenvolvesse uma sequência para a apresentação das ideias subjacentes ao conceito, antecipasse equívocos e conceitualizações ingênuas das crianças, construísse atividades úteis para desafiar essas concepções e planejasse experiências que permitissem às crianças fazer ligações entre ideias importantes (Leavy \& O’Loughlin, 2006).

Os artigos A.04 e A.05 enfatizam que há uma suposição de que o conteúdo a ser ensinado na escola primária (etapa inicial do ensino) já foi aprendido pelo professor em sua formação no ensino fundamental e médio e de que tal conteúdo é relativamente simples. Todavia, o que se aprende nessas etapas educativas não é adequado para se ensinar estatística de forma compreensiva para os estudantes, em outras palavras, o que o professor aprendeu quando estava estudando no ensino fundamental e médio não é o suficiente para que ensine estatística para os alunos do ensino primário.

Costa et al. (2016, p. 509), também alertam para esse equívoco no contexto brasileiro. Os autores afirmam "[...] é passível de questionamento a situação de que em um curso que forma o docente, os conhecimentos que os professores precisarão desenvolver em seus alunos estejam alicerçados apenas na educação que receberam durante o Ensino Fundamental e Médio”. E parece ter sido essa a formação destinada a professores do ensino elementar, principalmente para o ensino de matemática, disciplina que abarca os conteúdos de estatística na Educação Básica.

Em A.10, Witterholf, Goedhart e Suhre (2016) investigam como os professores holandeses em um processo de formação continuada, baseado na colaboração entre pares (redes de trabalho) projetam, implementam e avaliam novos métodos para o ensino de estatística para alunos do $7^{\circ}$ ano (correspondente ao $5^{\circ}$ ano brasileiro). Os participantes do estudo de A.10 comungavam a característica de terem pouca experiência com trabalhos envolvendo a pesquisa estatística ou com trabalhos de investigação ou por projetos. Para atender as especificidades das redes de trabalho, foram realizadas reuniões com duração de 50 a 90 minutos, destinadas a estabelecer objetivos, compartilhar experiências e desenvolver e avaliar em conjunto projetos de ensino.

Witterholf, et al (2016) mostram que a natureza das mudanças no conhecimento prático dos professores está atrelada às suas preocupações pessoais, que emergem da trajetória profissional. Alguns consideraram suas preocupações como desafios que estimulam a própria aprendizagem, enquanto outros experimentaram suas preocupações como um motivo para 
voltar aos métodos de ensino anteriores.

Os autores afirmam que a abordagem de rede foi positiva para o aprendizado dos professores, uma vez que a discussão e a reflexão criaram oportunidades para mudanças no conhecimento prático desses professores. O projeto de ensino de domínio da estatística, desenvolvido conjuntamente na rede de trabalho e implementado pelos professores, permitiu que refletissem sobre os seus conhecimentos e competências e que ampliassem seus conhecimentos práticos.

O trabalho colaborativo indicado por Roseth et al. (2008), em A.03, se aproxima das redes de trabalho apresentadas em A.10 e parecem adequadas para práticas de desenvolvimento profissional de professores. Segundo Roseth et al. (2008) o trabalho colaborativo promove uma visão compartilhada do ensino, o que é altamente benéfico para que se atinjam os objetivos fundamentais da educação. Do mesmo modo as redes de trabalho, como mostram Witterholf, et al (2016), são oportunas para a discussão, a troca de experiências e para a visão compartilhada do processo de ensino.

Os artigos A.11 e A.13 tratam do conhecimento sobre inferência estatística de futuros professores dos anos iniciais do ensino fundamental (contexto brasileiro). Os autores de A.11, Vetten, Schoonenboom, Keijzer, Oers (2018), mostram um estudo com 21 aspirantes a professores holandeses, que investigou o conhecimento sobre a inferência estatística informal durante a aplicação de uma curta intervenção em uma disciplina de educação matemática.

Em A. 13, os autores Justice, Zieffler, Huberty e DelMas (2018) envolveram professores secundários, estadunidenses e em exercício, e buscaram entender as percepções dos professores sobre o papel dos modelos estatísticos na inferência estatística e como esses propósitos percebidos afetam seu raciocínio sobre modelos estatísticos e inferência. Para a coleta de dados, Justice et al. (2018) realizaram entrevistas em que os participantes foram convidados a resolver alguns problemas, com uso do software TinkerPlots ${ }^{8}$.

Vetten et al. (2018) afirmam que as atividades propostas na intervenção foram oportunas para o desenvolvimento da consciência sobre a inferência e a necessidade de distinção entre uma amostra e uma população, e oportunas para o desenvolvimento do conhecimento sobre a variabilidade amostral e sobre amostra aleatória. Todavia, não foi observado nenhum avanço no desenvolvimento do conhecimento sobre tamanhos insuficientes de amostra e muitos dos participantes continuaram a favorecer a amostragem estratificada em detrimento da amostra

\footnotetext{
${ }^{8}$ O TinkerPlots é um software educacional comercial de análise de dados, desenvolvido por Konold \& Miller (2001), centrando-se no trabalho com crianças e jovens (Dias \& Santos Junior, 2018).
} 
aleatória, acreditando que a primeira seria mais representativa, sem considerar que para usar esse método amostral deve-se conhecer as proporções de características da população.

Os resultados de A.13 mostraram a tomada de decisão como o principal objetivo da modelagem estatística percebido pelos professores. Em outras palavras, os professores podem não ver a variação da modelagem como um propósito primário da modelagem estatística, em vez disso, substituem os dois outros propósitos: tomar uma decisão e replicar o processo de coleta de dados. Além disso, muitos dos professores não foram capazes de identificar claramente o papel de um modelo estatístico.

No artigo A.12, Gould, Bargagliotti e Johnson (2017) examinam como nove professores do ensino secundário dos Estados Unidos raciocinam com dados, partindo de atividades de modelagem e investigação estatística e com uso de big-data ${ }^{9}$. Os professores participavam de um curso de formação denominado Introdução à Ciência de Dados (IDS), o qual faz parte de um projeto maior, que tem por objetivo desenvolver habilidades computacionais com dados.

Então, o foco de A.12 está no papel da análise exploratória de dados. Os autores pontuam que a análise de dados envolve uma complexa interação entre análise, interpretação e questionamento, aspectos nos quais, nem todos os professores participantes do curso foram bem-sucedidos, o que sugere que outros estudos nessa linha são necessários para uma melhor compreensão do assunto.

O artigo A.14 se concentra no discurso de oito estudantes a professores alemães, ao comparar duas distribuições de uma variável numérica de um grande conjunto de dados reais com o TinkerPlots. Esse software foi observado em A.13, e foi considerado como uma boa ferramenta para o ensino e para a formação dos professores, pois, segundo os autores o software permite a exploração e a comparação entre conjuntos de dados. Frischemeier \& Biehler (2018), autores de A.14, afirmam que o desenvolvimento das habilidades de uso do TinkerPlots, para realizar análise de dados, é particularmente importante para futuros professores, principalmente se há a intenção de usar tecnologia em suas aulas. Aspecto que foi alcançado com o curso apresentado no artigo.

De maneira geral, os pesquisados em A.14, foram capazes de utilizar com sucesso o software em toda a tarefa proposta. Além disso, tanto o desenho do curso como o TinkerPlots, apoiaram os futuros professores na aprendizagem sobre comparações entre grupos de dados, promovendo o uso de conceitos apropriados de comparação ao passo que calculavam diferenças

\footnotetext{
9 Big-data são dados e informações amplas e multivariadas, incluem dados não numéricos e são coletados conforme determinado por um algoritmo ou por projetos experimentais tradicionais.
} 
entre duas distribuições em dados reais.

Entretanto, Frischemeier \& Biehler (2018), mencionam uma falha na interpretação dessas comparações, assim, sugerem que mais pesquisas sejam realizadas para estudo de como desenvolver competências de interpretação de resultados estatístico no contexto dos dados. Ainda apontam para algumas melhorias no curso por eles aplicado ou em práticas semelhantes, apontando para uma ressignificação no processo interpretativo dos dados. Esse ponto é observado em A.07 e A.08, em que Estrella (2016) e Lopes (2013) destacam que se deve ter atenção para a interpretação do contexto.

Os resultados e argumentos apresentados nos artigos de aplicação remetem aos conhecimentos necessários para o ensino de estatística proposto por Groth (2007), em A.01, sugerindo que a preparação para ensinar estatística, realmente, carece de uma maior atenção por parte das instituições formadoras nos cursos de formação docente inicial e continuada.

A exploração da compreensão dos professores sobre os conceitos estatísticos revelou que muitos conhecimentos, como das medidas de tendência central, da inferência estatística, dos tipos de amostra e representatividade, entre outros, precisam ser tratados nos cursos destinados a formar professores.

\subsection{Discussões Documentais}

Alguns artigos analisados nesta revisão se referem a estudos de currículo, metodologias e procedimentos de cursos para professores de estatística e foram classificados como discussões documentais (A.08, A.09 e A.15).

Em A.08, Lopes (2013) problematiza o desenvolvimento teórico e metodológico da disciplina de estatística na formação docente, com relatos de uma experiência vivenciada na Universidade da Geórgia no ano de 2008. O artigo A.08 é o único artigo de autoria brasileira, classificado entre os 15 mais relevantes e, apesar de tratar de um curso fora do país, a autora apresenta alguns estudos que tratam do contexto brasileiro, como os de Costa (2007), Costa e Nacarato (2011) e Costa e Pamplona (2011). Os autores citados por Lopes (2013) ressaltam as deficiências dos cursos de licenciatura em matemática, que acabam por enfatizar as questões técnicas e procedimentais da estatística, tratando-a como matemática aplicada.

Nesse sentido, a autora alerta para as diferenças entre estatística e matemática, como já se observou nos artigos A.01 e A.02, e para a necessidade de considerar a estatística como ciência de análise de dados. Para Lopes (2013) isso traz implicações para a formação docente, 
pois é necessário que sejam abordados nos cursos de formação aspectos inerentes a essa ciência, como a variabilidade e o contexto. Para a autora "a ciência estatística requer um tipo diferente de pensar, porque os dados não são apenas os números, eles são números com um contexto" (p. 905). A questão do contexto dos dados também foi discutida por Estrella (2016) em A.07, que enfatiza a interpretação do âmbito como meio para o entendimento da média e dos valores extremos (outliers).

Os argumentos apresentados, ainda se relacionam fortemente à proposta apresentada por Groth (2007) em A.01, que apresenta uma estrutura de conhecimentos matemáticos e nãomatemáticos necessários para ensinar estatística. Lopes (2013) ressalta que devem ser abordados nos cursos de licenciatura o quê, o porquê, o quando e, essencialmente, o como ensinar estatística. Deste modo, a autora enfatiza a necessidade de um pensamento de eficiência no ensino, ou seja, de como os professores podem ser mais eficazes em suas práticas para promover a aprendizagem estatística dos estudantes.

De acordo com Lopes (2013), a disciplina observada na Universidade da Geórgia traz esses elementos, partindo da concepção da estatística como ciência de análise de dados. A disciplina traz como metodologia central a resolução de problemas e o uso de recursos tecnológicos, promovendo atividades em grupo entre professores já atuantes e em formação inicial, aspecto destacado pela autora como diferencial na proposta da universidade. As colocações sobre a disciplina, apresentadas por Lopes (2013), dão indícios de que a disciplina em questão foi capaz de promover o desenvolvimento do pensamento estatístico e de auxiliar na compreensão de aspectos específicos, de como ensinar estatística para estudantes da Educação Básica.

No artigo A.09, Garfield e Everson (2009) descrevem um curso que prepara professores para o ensino de estatística para o Ensino Superior e para o Ensino Médio. Tal curso faz parte de um programa de pós-graduação em Educação Estatística. O currículo do curso é baseado na teoria de que bons professores de estatística precisam ser desenvolvidos, ao invés de serem treinados.

Para os autores um programa que tenha como intuito preparar os professores para ensinar estatística precisa estar alinhado com as diretrizes vigentes, assim como também, deve incluí-las em suas propostas. Desta maneira, o curso apresentado em A.09 fundamenta-se no framework GAISE. Outros artigos que tratam dos conhecimentos dos professores para ensinar estatística, analisados nesta revisão, também se baseiam fortemente nas orientações do GAISE (A.01, A.02, A.03, A.04 e A.05). 
Partindo desses pressupostos, o curso proposto por Garfield e Everson (2009) foi projetado de modo a oportunizar aos estudantes o entendimento sobre o GAISE e de como alinhar seu ensino com base nesse framework e, ainda, com a abordagem dos três componentes de conhecimentos propostos por Shulmam (1986) para o ensino, ou seja, do currículo, dos estudantes e de estatística (conteúdo).

Na descrição das tarefas realizadas por Garfield e Everson (2009), relacionadas às recomendações do GAISE (Franklin et al., 2007), observa-se que sempre houve a interação dos estudantes com os conteúdos estatísticos, para posteriormente análise de como poderiam ser tratados tais conceitos e se as metodologias empregadas poderiam ser implementadas em suas aulas. Além disso, há uma valorização do discurso estatístico, ou seja, momentos em que os estudantes são instigados a questionar uns aos outros e a dar respostas com argumentos baseados em dados.

Outro ponto fundamental apresentado em A.09 é o formato online do curso, que, segundo os autores, consegue manter a maioria dos elementos da versão presencial. É claro que algumas alterações foram necessárias, como as discussões entre os alunos/alunos e entre alunos/professor que passa a ser de forma escrita, o que requer atenção para a clareza ao se expressar, de modo que possam ser entendidos. A comunicação escrita, no entanto, pode ser útil, pensando-se em turmas presenciais em momentos nos quais não se consegue ouvir o que o outro está dizendo. O formato online apresentado pelos autores mostra que é possível formar professores com base no diálogo estatístico, mesmo que estes estejam fisicamente distantes.

Para a concretização de ações semelhantes, os autores sugerem cinco pontos: 1) reunir uma equipe de professores para discutir objetivos e atividades do curso; 2) ir além das necessidades do grupo a ser formado; ler artigos que não sejam apenas da comunidade de educação estatística; 4) oferecer um curso em que os participantes tenham oportunidade de ser alunos, sem fazer suposições sobre seus conhecimentos e 5) emprestar boas ideias de programas que já existem, modelar boas práticas de ensino, fornecer aos alunos materiais escritos para estudo e avaliação. Este último ponto, conforme Garfield e Everson (2009) é indicado por Moore (2005).

Essas sugestões apontam para a possibilidade de desenvolvimento de cursos de formação de professores para ensinar estatística, que podem se estender para outras etapas educativas. Ademais, os cursos podem fundamentar o desenvolvimento do conhecimento do conteúdo estatístico a partir das reflexões apontadas por Groth (2007) e Garfield e Gal (1999), que tratam de conhecimento de conteúdo e de avaliação em estatística, respectivamente. $\mathrm{O}$ 
primeiro ponto, sugerido pelos autores de A.06, Pfannkuch et al. (2010) expressa a questão da colaboração entre professores, aspecto discutido em A.03 por Roseth et al. (2008) e que pode ser muito útil, pensando-se em cursos em que professores com diferentes formações possam colaborar mutuamente para a concretização de ações mais efetivas de formação para o ensino de estatística.

Os autores do artigo A.15, Green et al. (2018), pesquisaram como os professores de matemática usam a estatística em suas próprias pesquisas. No estudo, analisaram uma amostra de nove trabalhos de pesquisa, realizados em salas de aula por professores que participam de um programa de mestrado para docentes do ensino fundamental. Green et al. (2018) observaram como os participantes planejaram os estudos e coletaram os dados; como analisaram esses dados e como interpretaram os resultados.

Os resultados mostram, embora de modo implícito, que há uma variedade de maneiras com as quais os professores usam as estatísticas para conduzir pesquisas e tomar decisões baseadas nos dados de suas pesquisas em sua sala de aula. Revelam, ainda, que os professores possuem um nível inicial do pensamento estatístico, que precisa ser melhor explorado.

Os autores afirmam que seus resultados sugerem que o conhecimento exigido dos professores sobre as medidas estatísticas é multifacetado, determinando componente pedagógico e conhecimento estatístico para a profissão docente. Em outras palavras, os professores precisam ter conhecimento pedagógico de estatística para o ensino, mas também precisam entender e usar estatísticas de maneiras significativas que lhes permitam tomar decisões informadas sobre seu currículo, instrução e práticas de ensino, assim como ressaltado nos artigos A.04, A.05, A.07, A.10 e A.11.

\section{Considerações Finais}

Com a leitura completa dos quinze artigos foi possível pontuar conhecimentos estatísticos necessários para os professores da Educação Básica, como sobre inferência estatística; métodos amostrais; raciocínio comparativo; propriedades da média e demais medidas de tendência central; análise exploratória de dados, entre outros. Tais conceitos aparecem nos artigos analisados como essenciais para a efetivação do ensino de estatística na Educação Básica, pois tratam de conhecimentos específicos sobre os quais os professores precisam ter domínio, tanto do conteúdo quanto pedagógico.

Em relação às metodologias, se observou a indicação de atividades que considerem o 
processo de investigação estatística do GAISE e do modelo PPDAC, a aprendizagem colaborativa, a modelagem estatística e o uso de softwares para a análise de dados. Sendo que as indicações para a condução do ensino são semelhantes para a elaboração de cursos, seguindo estruturas em que os professores em formação possam ter contato com atividades similares às quais aplicariam em suas salas de aula, ou seja, colocando os professores para interagir no processo de coleta, representação e análise de dados.

Muitos artigos criticam a formação inicial dos professores e afirmam a necessidade de cursos que fomentem o conhecimento do conteúdo e que, realmente, preparem o professor para o ensino de estatística. É consenso nos artigos analisados que ensinar estatística requer um conhecimento específico, que está relacionado a como construir significado para os dados, analisar o contexto, escolher os caminhos mais adequados, para responder a determinadas questões de pesquisa estatística, entre outros. Os autores, ainda indicam que esse conhecimento se dará com base em um preparo profissional que considere o conhecimento conceitual em detrimento do conhecimento procedimental.

\section{Agradecimentos}

Trabalho realizado com apoio da CAPES - Código de Financiamento 001.

\section{Referências}

Costa, A. (2007). A educação estatística na formação do professor de matemática. Dissertação de mestrado, Universidade São Francisco, Itatiba, SP, Brasil. Recuperado de: http://livros01.livrosgratis.com.br/cp069063.pdf

Costa, A., \& Nacarato, A. (2011). A estocástica na formação do professor de matemática: percepções de professores e de formadores. Bolema (Rio Claro), 24(39), 367-386. Recuperado em: http://ojsteste.biblioteca.unesp.br/index.php/bolema/article/view/5092

Costa, J. M., Pinheiro, N. A. M., \& Costa, E. (2016). A formação para matemática do professor de anos iniciais. Ciência \& Educação (Bauru), 22(2), 505-522. Recuperado de: http://www.scielo.br/pdf/ciedu/v22n2/1516-7313-ciedu-22-02-0505.pdf

Costa, W. N. G., \& Pamplona, A.S. (2011). Entrecruzando fronteiras: a Educação Estatística na formação de professores de Matemática. Bolema (Rio Claro), 24(40), 897-911.Recuperado em: https://bit.ly/2DMakX8

Estrella, S. (2016). Comprensión de la media por profesores de educación primaria en formación continua. Revista electrónica de investigación educativa, 18(1), 13-22. Recuperado de https://bit.ly/2PMrVDi 
Franklin, C., Kader, G., Mewborn, D., Moreno, J., Peck, R., Perry, M., \& Scheaffer, R. (2007). Guidelines for assessment and instruction in statistics education (GAISE) report: A preK12 curriculum framework. Alexandria, VA: American Statistical Association. Recuperado de https://www.amstat.org/asa/files/pdfs/GAISE/GAISEPreK-12_Full.pdf

Frischemeier, D., \& Biehler, R. (2018). Preservice teachers comparing groups with tinkerplots: an exploratory laboratory study3, 26. Statistics Education Research Journal, 17(1), 35-60. Recuperado de http://www.stat.auckland.ac.nz/serj

Garfield, J., \& Everson, M. (2009). Preparing Teachers of Statistics: A Graduate Course for Future Teachers. Journal of Statistics Education, 17(2), null-null. https://doi.org/10.1080/10691898.2009.11889516-x

Garfield, J. B., \& Gal, I. (1999). Assessment and Statistics Education: Current Challenges and Directions. International Statistical Review, 67(1), 1-12.

Gould, R., Bargagliotti, A., \& Johnson, T. (2017). An Analysis of Secondary Teachers' Reasoning with Participatory Sensing Data. Statistics Education Research Journal, 16(2), 305-334. Recuperado de https://eric.ed.gov/?id=EJ1162357

Green, J. L., Smith, W. M., Kerby, A. T., Blankenship, E. E., Schmid, K. K. \& Carlson, M. A. (2018). Introductory statistics: preparing in-service middle-level mathematics teachers for classroom research. Statistics Education Research Journal, 17(2), 216-238. Recuperado de http://www.stat.auckland.ac.nz/serj

Groth, R. E. (2007). Toward a Conceptualization of Statistical Knowledge for Teaching. Journal for Research in Mathematics Education, 38(5), 427-437. https://doi.org/10.2307/30034960

Jacobbe, T. (2012). Elementary school teachers' understanding of the mean and median. International Journal of Science and Mathematics Education, 10(5), 1143-1161. https://doi.org/10.1007/s10763-011-9321-0

Justice, N., Zieffler, A., Huberty, M. D., \& delMas, R. (2018). Every rose has its thorn: secondary teachers' reasoning about statistical models. ZDM, 50(7), 1253-1265. https://doi.org/10.1007/s11858-018-0953-1

Leavy, A., \& O’Loughlin, N. (2006). Preservice Teachers Understanding of the Mean: Moving Beyond the Arithmetic Average. Journal of Mathematics Teacher Education, 9(1), 53-90. https://doi.org/10.1007/s10857-006-9003-

Lopes, C. E. (2013). Educação Estatística no Curso de Licenciatura em Matemática $</ \mathrm{b}>$. Bolema - Boletim de Educação Matemática, 27(47), 901-915. Recuperado de https://bit.ly/2vxRy1h

Moore, D. (2005). Preparing Graduate Students to Teach Statistics: Introduction. The American Statistician, 59, 1-3.

Pagani, R. N., Kovaleski, J. L., \& Resende, L. M. (2015). Methodi Ordinatio: a proposed 
methodology to select and rank relevant scientific papers encompassing the impact factor, number of citation, and year of publication. Scientometrics, 105(3), 2109-2135. https://doi.org/10.1007/s11192-015-1744-x

Pfannkuch, M., Regan, M., Wild, C., \& Horton, N. J. (2010). Telling Data Stories: Essential Dialogues for Comparative Reasoning. Journal of Statistics Education, 18(1), null-null. https://doi.org/10.1080/10691898.2010.11889479

Roseth, C. J., Garfield, J. B., \& Ben-Zvi, D. (2008). Collaboration in Learning and Teaching Statistics. Journal of Statistics Education, 16(1), null. https://doi.org/10.1080/10691898.2008.11889557

Shulman, L. S. (1986). Those who unsderstand: knowledge growth in teaching. Educational Researcher, 15(2), 4-14.

Vetten, A. de, Schoonenboom, J., Keijzer, R., \& van Oers, B. (2018). The development of informal statistical inference content knowledge of pre-service primary school teachers during a teacher college intervention. Educational Studies in Mathematics, 99(2), 217-234. https://doi.org/10.1007/s10649-018-9823-6

Wild, C.J. \& Pfannkuch (1999). Statistical Thinking in Empirical Enquiry (with discussion). International Statistical Review, 67(3), 223-265.

Witterholt, M., Goedhart, M., \& Suhre, C. (2016). The impact of peer collaboration on teachers' practical knowledge. European Journal of Teacher Education, 39(1), 126-143. https://doi.org/10.1080/02619768.2015.1109624 